\title{
Conversion disorder: advances in our understanding
}

\author{
Anthony Feinstein MD PhD
}

I n 1968, the word hysteria, present for more than two millennia and used to describe atypical signs and symptoms that did not conform to established diseases, disappeared from the Diagnostic and Statistical Manual of Mental Disorders (DSM) of the American Psychiatric Association's classification of mental disorders. ${ }^{1}$ Critics had argued that the condition lacked validity and that it owed its longevity to tradition. Moreover, the term was pejorative with its fallacious association to uterine pathology. Abolishing the word did not, however, remove a disorder, nor did it lead to fresh insights as to the condition's cause, given the largely descriptive content of the DSM. It also did not adequately solve the taxonomy conundrum.

\section{The disappearance of hysteria}

A new category, the somatoform disorders, was created to encompass some of the conditions displaced by the changing nomenclature. This broad rubric was split into six conditions (see Box 1), two of which are quite relevant to the discarded all-embracing category of hysteria.

The first condition is somatization disorder (also called Briquet syndrome), which refers to a diverse symptomatology involving multiple body systems (the fourth edition of the DSM [DSMIV] spells out the minimum number of symptoms per system: four discrete pain symptoms, two gastrointestinal symptoms, one sexual or reproductive symptom and one pseudoneurological symptom that must have begun before 30 years of age, for which treatment was sought or that caused the patient substantial impairment in functioning in social or occupational settings). ${ }^{1}$ After appropriate investigation, each of the symptoms cannot be fully explained by a known medical condition or the direct effects of a substance (either illicit or prescribed).

The second condition, conversion disorder, best fits the clinical description of patients who received treatment from such luminaries as Charcot and Freud during the last decades of the 19th century. Here, the signs and symptoms are limited to those that suggest a neurologic condition. Once more, the presentation cannot be explained using appropriate investigations, nor can they be

attributed to the patient's use of substances or participation in culturally sanctioned behaviours (e.g., trances during religious ceremonies). Four types of conversion disorder are specified: those with motor symptoms or deficits, those with sensory symptoms or deficits, those with pseudoseizures and a mixed presentation.

Hysteria, however, cast a wider net than the newly minted somatoform category. To capture the outliers, one must turn to another category of the DSM-IV, the dissociative disorders (see Box 1) - specifically, dissociative amnesia. The predominant disturbance is one or more episodes during which the patient is unable to recall important personal information that is usually of a traumatic or stressful nature.

Hysteria's dismemberment was arbitrary and has led to confusion in the two main systems for classifying mental illness: the DSM-IV and the International Statistical Classification of Diseases and Related Health Problems, 10th revision (ICD-10). ${ }^{2}$ In the latter, for example, conversion disorder appears under the broad category of dissociative (conversion) disorders. This difference, however, is essentially semantic. Looking to the future, the working groups for the fifth edition of the DSM have proposed yet another radical revision to the taxonomy. Although the essential diagnostic features of conversion disorder will remain unchanged, the condition is to be renamed "functional neurological symptoms."

Conversion disorder may be broadly defined as the presence of neurologic symptoms in the absence of a neurologic diagnosis, or when a

\section{- Ker pOINTS}

- Conversion disorder refers to a condition with neurologic symptoms that cannot be fully explained by neurologic disease.

- Symptoms of conversion disorder are not feigned or deliberately produced; rather, they reflect the "conversion" of underlying emotional distress into physical (neurologic) symptoms.

- Functional magnetic resonance imaging has shown cerebral correlates of clinical signs.

- Although there is general agreement that treatment should address the underlying emotional distress, clinical trials are few and results need to be replicated.

- Preliminary data from cognitive behaviour therapy and transcranial magnetic stimulation interventions are promising. 
neurologic diagnosis exists, it does not fully account for all of the patient's symptoms. The purpose of this review is to examine the classification, epidemiology and theories as to the causes of conversion disorder, as well as the issues related to the condition's diagnosis and management. The paper will also focus on advances in magnetic resonance imaging (MRI) that complement long-held psychoanalytic beliefs concerning the notion of primary gain underpinning the pathogenesis of conversion symptoms, i.e., the "conversion" of emotional distress into a presentation of physical illness; in this case, neurologic symptoms.

\section{Literature review}

There is extensive medical literature devoted to hysteria. A Medline search going back to 1968, the year in which the second edition of the DSM was published, retrieved more than 4000 articles on hysteria. For this focused update on conversion disorder, the term "conversion disorder" was added to the search. This additional search term reduced the number of articles to 352 . The search was further narrowed by restricting the language of the articles to English. Pediatric studies and case reports were also excluded, unless they pertained to brain imaging. With these criteria as a baseline, the following selective terms (corresponding to the subcategories addressed in this review) were added to the search: epidemiology, which returned 41 articles; etiology, returning 123 articles; misdiagnosis, with 7 articles; brain imaging, which returned 12 articles; and treat-

Box 1: Diagnostic categories encompassing the former category of "hysteria," as defined in the Diagnostic and Statistical Manual of Mental Disorders, 4th ed. ${ }^{1}$

Somatoform disorders

- Somatization disorder*

- Conversion disorder*

- Undifferentiated somatoform disorder

- Pain disorder

- Hypochondriasis

- Body dysmorphic disorder

Dissociative disorders

- Dissociative amnesia*

- Dissociative fugue

- Dissociative identity disorder

- Depersonalization disorder

- Dissociative disorder not otherwise specified (this includes the Ganser syndrome)

*Diagnoses particularly relevant to the former diagnostic category of "hysteria." ment, which returned 21 articles. Taking into account overlap between articles, as well as their relevance, the references selected here are a distillation of this focused search.

\section{How common is conversion disorder?}

Prevalence rates vary according to the composition of the population studied and whether the authors reported the frequency of conversion symptoms or the diagnosis of the syndrome. Studies have estimated that $20 \%-25 \%$ of patients in a general hospital setting have individual symptoms of conversion, ${ }^{4}$ and $5 \%$ of patients in this setting meet the criteria for the full syndrome. ${ }^{5}$ Not surprisingly, percentages increase in a neurologic setting. One in five outpatients seen in a neurology clinic may have symptoms that cannot be attributed to neurologic disease. ${ }^{6} \mathrm{~A}$ study of 100 consecutive patients newly admitted to a neurology ward found that $14 \%$ had no objective evidence of neurologic disease. ${ }^{7}$ Data from psychiatric services give a different picture where individual symptoms, considered virtually ubiquitous, ${ }^{8}$ translate into a lifetime prevalence of 23/100 000 for the full disorder. ${ }^{9}$ This corresponds to the rate of $11-22 / 100000$ found in a psychiatric care registry of the general population. ${ }^{10}$ Earlier estimates of $1 \%$ in a sample of women who had recently given birth ${ }^{11}$ and $3 \%$ $7 \%$ in general out-patient clinics ${ }^{8,12}$ should be viewed as grossly inflated, given selection bias, the small sizes of the samples and the absence of structured interviews. The considerable variability in prevalence according to clinical setting suggests a heightened diagnostic vigilance from clinicians where appropriate.

There is broad agreement that conversion disorder is more common in women, with an age of onset across the lifespan (unlike somatization disorder, which predominantly affects young women).${ }^{13}$ However, the observation made over a quarter of a century ago that the disorder was found more frequently among people who lived in rural areas or who had lower levels of education or belonged to a lower socioeconomic class $^{10}$ deserves a fresh look rather than dogmatic repetition as fact.

\section{What causes conversion disorder?}

The DSM-IV largely shies away from theories as to the causes of disorders, but relents a little when it comes to the somatizing patient. Conversion disorder is attributed to conflicts or recent stressors. These precipitating events are also 
implicit in the criteria for diagnosing dissociative amnesia, given the stated association with emotionally traumatic events. This channeling, or conversion, of emotional arousal to physical symptoms is termed the primary gain. Secondary gain refers to the external benefits that may be derived as a result of having symptoms. For example, the patient whose sudden onset of paresis (primary gain) causes his or her spouse to stay in an otherwise failing relationship (secondary gain).

Common to all of these disorders is the principle that signs and symptoms are not deliberately or consciously produced. Indeed, the idea that unconscious (involuntary) processes were the underpinning of the psychopathology was instrumental to the development of Sigmund Freud's theory of psychoanalysis.

Although the understanding of conversion disorder as defined by the DSM-IV reflects the influence of psychoanalysis, other theories have also been advanced to account for conversion symptoms. Learning theory posits that the environment shapes and influences behaviour. Broadly stated, behaviours that result in positive consequences (positive reinforcement) or that remove aversive stimuli (negative reinforcement) are repeated, and behaviours that result in negative consequences (e.g., punishment) are avoided. Should these behaviours become maladaptive, a conversion disorder may, in theory, ensue. For example, physical abuse - an aversive stimulus might trigger attention-demanding behaviours such as pseudoseizures or aphonia.

An alternative explanation comes from sociocultural theorists. Among certain cultures, the expression of intense emotion is not sanctioned. Physical symptoms, considered more acceptable, become the conduit through which the emotionally distressed person communicates his or her feelings or troubled thoughts.

\section{How is the diagnosis made?}

The diagnosis of a possible conversion disorder should be considered when neurologic signs are atypical, do not conform anatomically or are at odds with the results of clinical investigations. In such a situation, excessive investigations carry an iatrogenic risk and may prolong a patient's symptoms. The absence of a clearly identifiable stressor is not uncommon; such an absence does not invalidate the diagnosis and clinicians should not be dissuaded from further psychological inquiry. The diagnostic criteria for conversion disorder are shown in Box 2.

If a clinician concludes that a patient's symptoms are being produced intentionally (though such a distinction is not always straightforward), then the diagnosis changes to either factitious disorder or malingering. Factitious disorder (also known as the Munchhausen syndrome) refers to individuals who deliberately feign symptoms (physical, psychological or both) for the express purpose of adopting the role of a person with an illness. This differs from malingering, in which the motivation behind the deception is some external incentive, such as monetary gain, evading criminal prosecution or avoiding military service.

\section{Diagnostic accuracy}

In 1965, an influential follow-up study reported that $33 \%$ of patients diagnosed with hysteria about 10 years earlier subsequently received a different diagnosis that could explain their initial symptoms. ${ }^{14}$ Data such as these, when replicated, contributed to the demise of hysteria as a diagnostic category. However, follow-up studies done after 1970 have, on average, reported a significantly lower rate of misdiagnosis of conversion symptoms, namely in the order of $0.4 \%$ $4 \% .^{15,16}$ This difference may be explained by improvements in the quality of the follow-up studies and, to a lesser extent, the advances made in neuroimaging techniques.

\section{What can advances in neuroscience tell us about conversion disorder?}

With ideas like primary and secondary gain, and the posited relationship between the development of symptoms and the presence of some external stressor, conversion disorder has long been viewed as the quintessential psychological disorder. Psychosocial causes were deemed more

\section{Box 2: Diagnosing conversion disorder ${ }^{1}$}

- The patient must have at least one symptom or deficit that affects his or her voluntary motor or sensory function, suggesting a neurologic or other medical condition. Patients may also present with seizures or convulsions, or with a mixture of symptoms or deficits.

- Psychological factors appear to be associated with the symptom or deficit. For example, symptoms may start or worsen after a stressor such as a conflict.

- The patient's symptom or deficit causes him or her to feel distress or have difficulties when engaged in important activities (e.g. social, occupational). The symptom or deficit may also warrant medical investigation.

- Other diagnoses (e.g. factitious disorder or malingering) should be considered if the physician suspects that the patient is feigning or producing the symptoms or deficits intentionally.

- After appropriate investigation, no explanation fully accounts for the symptom or deficit.

- The symptom or deficit should not be better explained by another mental disorder and does not occur during the course of somatization disorder (or Briquet syndrome). The symptom or deficit should not be limited to pain or sexual dysfunction. 
important than cerebral causes, a theory that was bolstered by long-held, increasingly outmoded notions of a functional-organic divide. Advances in neuroscience are slowly changing this view, but the challenge is considerable given the heterogeneity of clinical expression, frequent presence of comorbidities such as anxiety and depressive disorders and the role of psychosocial stressors as putative precipitating factors and, at times, perpetuating factors.

There is a paucity of data from structural imaging of the brains of people with conversion disorder. One study showed reduced volumes of the right and left basal ganglia and right thalamus relative to people without the disorder. ${ }^{17}$

Emerging literature on the functional imaging of the brain is more informative. Sample sizes are modest (single case studies predominate), experimental paradigms differ, and participants, mostly female and young to middleaged, vary in their presentation (sensory, motor or mixed sensory and motor symptoms). Despite these limitations, however, some consistent results are being seen.

In a study of unilateral sensory loss thought to be a conversion symptom, patients had a vibratory stimulus applied first to their sensate region, then to their anesthetic side. Data from a functional MRI study showed contralateral somatosensory activation when the stimulus was applied to the sensate region, as expected, but no such activation when the stimulus was applied to the anesthetic side (Figure 1). ${ }^{18}$ Instead, the stimulus applied to the anaesthetic side activated regions in the patients' orbitofrontal and anterior cingulate regions. Similar

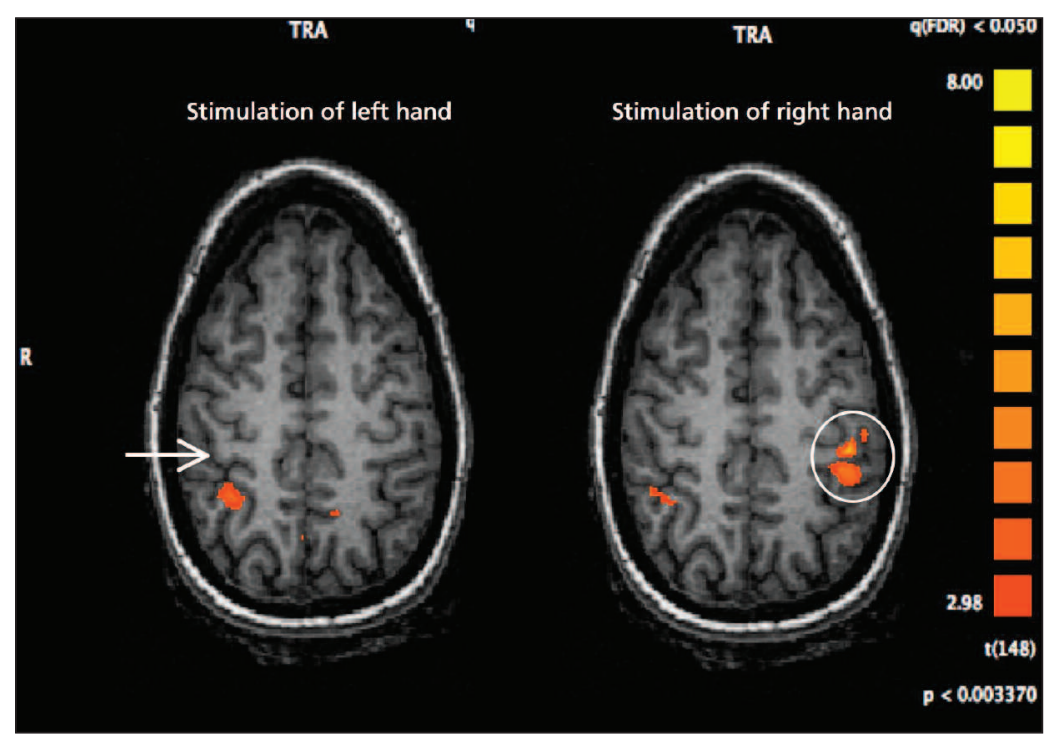

Figure 1: Functional magnetic resonance image showing somatosensory activity evoked by stimulation in a patient with sensory conversion disorder affecting the left hand. When the patient's left hand was stimulated, no activity was seen in the primary somatosensory cortex (arrow). However, increased activity was seen in this area of the brain when the patient's right hand was stimulated (circle). results, including anciliary activation in the basal ganglia, have emerged from other studies using functional MRI and single-photon emission computed tomography (SPECT) scans to investigate motor conversion symptoms. ${ }^{19,20}$ The association between conversion symptoms and activity in the orbitofrontal and cingulate regions is informative because these regions are important components of the neural networks regulating emotion and the expression of that emotion, i.e., a person's affect.

These results suggest that patients with conversion disorder have an abnormal pattern of cerebral activation in which limbic areas (or areas richly connected to the limbic system) override the activation of the motor and sensory cortices. Precisely how this happens is unclear, but one theory holds that specific regions of the cingulate cortex may function in a mutually exclusive way. A mechanism called "reciprocal inhibition" allows each region to shut off the other during the processing of information. This is relevant to conversion disorder in that the caudal segment, responsible for willed action, may be deactivated or suppressed by the pregenual anterior cingulate cortex as it processes intense emotion. ${ }^{21}$

Patients with repressed (unwanted) emotional memories (what the DSM terms dissociative amnesia) have a functional MRI pattern of regional cerebral deactivation and ancillary activation that complements the data on conversion disorder. Neural activity in the hippocampus (a repository of memories) is suppressed by activation in a network rich in frontal (i.e., dorsolateral and ventrolateral prefrontal cortices and the anterior cingulate gyrus) connectivity. ${ }^{22}$ It is to be expected that regional brain specificity would differ between the data from functional MRI studies of patients with conversion disorder and patients with dissociative amnesia, given the differences in phenomenology. Of greater importance is that, in both conditions, the discrete neural networks involved in processing emotion and executive control can suppress regions associated with a plethora of other functions (e.g., motor, sensory, memory, vision). This bolsters the validity of the construct underpinning how atypical symptoms, irrespective of their phenotype, may arise. These explanations do not necessarily refute current psychological theories as outlined by the DSM-IV. Rather, they provide a complementary cerebral model to account for the development of symptoms using the notion of primary gain. What the imaging data cannot answer, however, is why a particular symptom manifests.

At present, functional MRI technology remains largely experimental and is not yet used in routine clinical practice. In time, this is likely to change. 


\section{Which treatments are effective?}

Given that prognosis in conversion disorder is linked to the duration of symptoms, it behooves the patient's physician (Box 3) to rule out neurologic causes as quickly as possible and to refer the patient to a psychiatrist (or a psychologist, depending on the treatment provided). Treatment can be challenging and empirical data are lacking. Randomized controlled trials have shown that cognitive behavioural therapy is effective and is the treatment of choice for somatoform disorders. ${ }^{23,24}$ However, the only data on cognititive behavioural therapy for conversion disorder is from a single pilot study that reported some benefit. ${ }^{25}$

Data on psychoanalytic treatment are sparse and limited to isolated case reports, which is surprising given the strong historical and causal links between conversion-type symptoms and classic Freudian theory. Results from two trials of hypnosis have been contradictory ${ }^{26,27}$ Preliminary findings from studies with small sample sizes suggest that antidepressant medication, ${ }^{28}$ behavioural therapy, ${ }^{29}$ paradoxical intention ${ }^{30}$ and transcranial magnetic stimulation ${ }^{31}$ may be effective, but these results need to be replicated. From a theoretical standpoint, the data on transcranial magnetic stimulation are intriguing. Transcranial magnetic stimulation appears to have the ability to "turn on" regions of the brain, such as the motor and sensory cortices, when they have been_deactivated by unconsciously driven limbic activity. This observation dovetails with the functional MRI data elucidating the functional neuroanatomy of conversion. ${ }^{32}$

When treating conversion disorder, clinicians should be aware of the possibility of psychiatric comorbidity. For example, about one third of patients with conversion disorder also have major depression. ${ }^{8}$ Successfully treating the depression may lead to improvement in, or resolution of, the conversion disorder, given the removal of the primary gain that underpins the conversion reaction. In the presence of often complex symptoms and causes, a multidisciplinary approach to treatment using pharmacotherapy, psychotherapy, physiotherapy and intervention by a social worker, where necessary, is intuitively compelling; however, empirical data supporting such an approach are absent.

Good prognostic signs include an acute onset and short duration of symptoms, a clearly identifiable stressor, access to a therapist and a psychologically minded patient. A recent study of how neurologists view conversion disorder showed that some clinicians fail to understand their patients psychologically and may not distinguish a patient's symptoms from feigning. How these attitudes influence prognosis is not known. ${ }^{33}$

\section{Box 3: The primary care physician and conversion disorder}

Given that multiple health care providers are often involved in the management of this disorder, good communication between treating clinicians is required to maintain a consistent approach to treatment. The primary care physician can play a pivotal role in preventing the patient from seeking multiple second opinions.

Other key aspects for the family physician include:

- considering the possibilities of conversion disorder when a patient's neurologic signs are atypical, do not conform anatomically or are at odds with the results of investigations;

- minimizing investigations (excessive investigations may prolong symptoms);

- considering early referral to a neurologist if the diagnosis is unclear;

- quickly referring patients with conversion disorder to a psychiatrist or psychologist for further assessment and treatment (prognosis is linked to the duration of symptoms);

- monitoring for psychiatric comorbidities, such as major depression, which should be treated;

- watching for a recurrence or change in symptoms and referring back to the treating psychiatrist or psychologist should either occur.

For most patients, acute symptoms of conversion disorder resolve within weeks, but 20\%-25\% of patients may have recurring symptoms within a year, often in association with a stressful event.' The outcome is very different among patients with conversion disorder that has been ongoing for longer than one year. For these patients, the persistence of symptoms is common and is often linked to significant psychiatric comorbidity ${ }^{34}$

\section{Conclusions}

While taxonomists continue to fiddle, advances in neuroimaging are shedding new light on the origins of what have previously been seen as unexplained neurologic symptoms. However, insights into the pathogenesis of these symptoms continue to outstrip empirically derived data on their treatment. Future clinical trials with cognitive behavioural therapy and transcranial magnetic stimulation are needed.

\section{References}

1. American Psychiatric Association. Diagnostic and statistical manual of mental disorders. 4th ed. Washington (DC): American Psychiatric Association; 1994

2. World Health Organization. The ICD-10 classification of mental and behavioral disorders. Geneva (Switzerland): The Organization; 1992.

3. American Psychiatric Association. Conversion disorder. Arlington (VA): The Association; 2011. Available: www. dsm5.org/proposedrevisions/pages/proposedrevision.aspx?rid=8 (accessed 2011 Apr. 13).

4. Engl GL. Conversion symptoms. In: McBride CM, editor. Signs and symptoms: applied pathologic physiology and clinical interpre tation. 5th ed. Philadelphia (PA): JB Lippincott; 1970. p. 650-68.

5. Folks DG, Ford CV, Regan WM. Conversion symptoms in a general hospital. Psychosomatics 1984;25:285-89.

6. Mace CJ, Trimble MR. 'Hysteria', 'functional' or 'psychogenic'? A survey of British neurologists' preferences. J R Soc Med 1991; 84:471-5.

7. Ewald H, Rogne T, Ewald $\mathrm{K}$, et al. Somatization in patient newly admitted to a neurology department. Acta Psychiatr Scand 1994;89:174-9. 
8. Woodruff RA, Clayton PJ, Guze SB. Hysteria: an evaluation of specific diagnostic criteria by the study of randomly selected psychiatric clinic patients. Br J Psychiatry 1969;115:1243-8.

9. Tomasson K, Kent D, Coryell W. Somatization and conversion disorders: co-morbidity and demographics at presentation. Acta Psychiatr Scand 1991;84:288-93.

10. Stefánsson JG, Messina JA, Meyerowitz S. Hysterical neurosis conversion subtype: clinical and epidemiological considerations. Acta Psychiatr Scand 1976;53:119-38.

11. Farley J, Woodruff RA. Guze SB. The prevalence of hysteria and conversion symptoms. Br J Psychiatry 1968;114:1121-5.

12. Stefanis C, Markidis M, Christodoulou G. Observations on the evolution of the hysterical symptomatology. Br J Psychiatry 1976;128:269-75.

13. Owens C, Dein S. Conversion disorder: the modern hysteria. Adv Psychiatr Treat 2006;12:152-7.

14. Slater E. Diagnosis of hysteria. BMJ 1965;1:1395-9.

15. Stone J, Carson A, Duncan R, et al. Symptoms 'unexplained by organic disease' in 1144 new neurology out-patients: how often does the diagnosis change at follow-up? Brain 2009;132:2878-88.

16. Stone J, Smyth R, Carson A, et al. Systematic review of misdiagnosis of conversion symptoms and "hysteria." BMJ 2005;331:989.

17. Atmaca M, Aydin A, Tezcan E, et al. Volumetric investigation of brain regions in patients with conversion disorder. Prog Neuropsychopharmacol Biol Psychiatry 2006;30:708-13.

18. Ghaffar O, Staines R, Feinstein A. Functional MRI changes in patients with sensory conversion disorder. Neurology 2006;67: 2036-8.

19. Marshall JC, Halligan PW, Fink GR, et al. The functional neuroanatomy of a hysterical paralysis. Cognition 1997;64:B1-B8.

20. Vuilleumier P, Chichério C, Assal F, et al. Functional neuroanatomical correlates of hysterical motor loss. Brain 2001;124:1077-90.

21. van Beilen M, Vogt B, Leenders K. Increased activation in cingulate cortex in conversion disorder: What does it mean? J Neurol Sci 2010;289:155-8

22. Anderson MC, Ochsner KN, Kuhl B, et al. Neural systems underlying the suppression of unwanted memories. Science 2004;303:232-5.

23. Kroenke K. Efficacy of treatment of somatoform disorders: a review of randomized controlled trials. Psychosom Med 2007; 69:881-8.
24. Sumathipala A. What is the evidence for the efficacy of treatments for somatoform disorders? A critical review of previous intervention studies. Psychosom Med 2007;69:889-900.

25. Goldstein LH, Deale AC, Mitchell-O'Malley SJ, et al. An evaluation of cognitive behavior therapy as a treatment for dissociative seizures: a pilot study. Cogn Behav Neurol 2004;17:41-9.

26. Moene FC, Spindhoven P, Hoogduin KA, et al. A randomized controlled clinical trial on the additional effect of hypnosis in a comprehensive treatment programme for in-patients with conversion disorder of the motor type. Psychother Psychosom 2002; 71:66-76.

27. Moene FC, Spindhoven P, Hoogduin KA, et al. A randomized controlled clinical trial of a hypnosis based treatment for patients with conversion disorder, motor type. Int J Clin Exp Hypn 2003; 51:29-50.

28. Voon V, Lang AE. Antidepressant treatment outcomes of psychogenic movement duisorders. J Clin Psychiatry 2005;66: 1529-34.

29. Shapiro AP, Teasell RW. Behavioral interventions in the rehabilitation of acute v. chronic non-organic (conversion/factitious) motor disorders. Br J Psychiatry 2004;185:140-6.

30. Ataoglu A, Ozcetin A, Icmeli C, et al. Paradoxical therapy in conversion reaction. J Korean Med Sci 2003;18:581-4.

31. Schönfeldt-Lecuona C, Conneman BJ, Viviani R, et al. Transcranial magnetic stimulation in motor conversion disorder: a short case series. J Clin Neurophysiol 2006;23:472-5.

32. Feinstein A. Editorial commentary on psychogenic aphonia: spectacular recovery after motor cortex transcranial magnetic stimulation. J Neurol Neurosurg Psychiatry 2009;80:4.

33. Kanaan R, Armstrong D, Barnes P, et al. In the psychiatrist's chair: how neurologists understand conversion disorder. Brain 2009 132:2889-96.

34. Feinstein A, Stergiopoulos V, Fine J, et al. A prospective study of psychiatric outcome in patients with a psychogenic movement disorder. J Neuropsychiatry Neuropsychol Behav Neurol 2001; 14:169-76.

Affiliation: Dr. Anthony Feinstein is with the University of Toronto and the Department of Psychiatry, Sunnybrook Health Sciences Centre, Toronto, Ont. 